\title{
Study on Dipole Moments of Macromolecules I. Apparatus for Dielectric Measurements and Dipole Moment of Poly( $p$-methoxystyrene)
}

\author{
Noriko Yamaguchi, Mari SATo, and Mikiko ShIMA \\ Tokyo Woman's Christian University, \\ Zempukuji, Suginami-ku, Tokyo 167, Japan
}

(Received May 21, 1987)

\begin{abstract}
A three-terminal cell for the dielectric measurement of liquid was made and the cell capacitance was determined using a capacitance bridge. The accuracy of the dielectric constant measurement with the apparatus was examined by measuring standard materials such as benzene and cyclohexane. The mean square dipole moments of poly ( $p$-methoxystyrene) in benzene were determined by accurate measurements of dielectric constants on the polymer dilute solutions and the values were estimated according to two procedures, i.e., one based on Debye and HalverstadtKumler equations (DHK method) and the other on Guggenheim and Smith equations (G-S method). The average dipole moments per repeat unit $\mu$ were obtained as $1.214\left(25^{\circ} \mathrm{C}\right)$ and 1.221 $\left(40^{\circ} \mathrm{C}\right)$ by the DHK method and $1.204\left(25^{\circ} \mathrm{C}\right)$ and $1.209\left(40^{\circ} \mathrm{C}\right)$ by the G-S method (in debye unit). The slight disagreement in $\mu$ values obtained by two different procedures was interpreted as arising from differences in assumptions used in the DHK method and the G-S method.
\end{abstract}

KEY WORDS Dipole Moment / Dielectric Constant / Molecular

Polarization / Electronic Polarization / Atomic Polarization / Molecular

Refraction / Poly( $p$-methoxystyrene) / Dielectric Cell / Dilute Solution /

Dipole moment is a configurationdependent property of polymer chains carrying polar groups in their monomer units and the observed dipole moment is the statistical average of molecular conformations. Theory predicts that the dipole moments of polymer molecules cannot be affected greatly by longrange excluded volume interactions when the molecules have the dipole components perpendicularly attaching to the chain axis. ${ }^{1,2}$ The probable absence of the effect of the excluded volume on dipole moments, in contrast with its pronounced effect on chain dimensions, is of great advantage in investigating the influence of intramolecular interactions on the conformation of polar polymers.

We have been studying since early in the 1960's the dipole moments $\mu$ of polar vinyl polymers to investigate configurational prop- erties such as the stereochemical structure of the vinyl chains and the monomer sequence distribution in the copolymers. ${ }^{3-10}$ Since the conformational analysis of the polymer chains was developed by Flory et al. on the basis of a rotational isomeric model (RIS model), ${ }^{11}$ a number of studies on the dipole moments of various polymers have been carried out theoretically and experimentally. The dipole moment ratio, $D_{\mathrm{r}}=\left\langle\mu^{2}\right\rangle / \mathrm{x}^{2}$, and the temperature coefficient, $\mathrm{d} \ln \left\langle\mu^{2}\right\rangle / \mathrm{d} T$, are important parameters in the investigation of the conformational properties of a polymer, where $\left\langle\mu^{2}\right\rangle$ is the mean square dipole moment of the polymer with $\mathrm{x}$ bond dipoles of magnitude $m$ in the chains. The quantities of $D_{\mathrm{r}}$ and $\mathrm{d} \ln \left\langle\mu^{2}\right\rangle / \mathrm{d} T$ for polystyrene derivatives in various solvents were reported by many workers..$^{6,9,10,12-16}$ Theoretical calculations of 
these quantities were carried out for $\operatorname{poly}(p$ chlorostyrene) (PClSt) by Mark and Saiz on the basis of the RIS model. ${ }^{17}$ There is, however, much scattering in the experimentally observed values of $D_{\mathrm{r}}$ and $\mathrm{d} \ln \left\langle\mu^{2}\right\rangle / \mathrm{d} T$ and conclusive results have not been obtained. $D_{\mathrm{r}}$ values for PCISt at $25^{\circ} \mathrm{C}$ range from 0.4 to 0.7 ( $m=1.90$ debye was used) in which there is no obvious correlation with the solvent type employed in the measurements; the negative values of $-8 \times 10^{-3} \sim-1.6 \times 10^{-3}$ were observed for $\mathrm{d} \ln \left\langle\mu^{2}\right\rangle \mathrm{d} T$ of PCISt, ${ }^{9,12,13,16}$ while an increasing tendency of $\mu$ with temperature and/or an inflection of the temperature dependence at a certain temperature has been reported. ${ }^{6,12,14,16}$ These facts do not permit a quantitative comparison with theoretical results.

In this paper, we report a new dielectric apparatus with a capacitance bridge and a three-terminal cell prepared to improve the accuracy of dielectric measurements and present some data on the dielectric constants of organic solvents such as benzene and cyclohexane. We then report the dipole moments determined by two procedures for $\operatorname{poly}(p$ methoxystyrene) in benzene and discuss the underlying assumptions for determining the dipole moments. $\mu$ is usually estimated according to the conventional procedure known as Debye's method, ${ }^{18}$ by measuring the dielectric constant and the density of polymer solutions. On the other hand, in many recent papers $\mu$ is determined by the use of the GuggenheimSmith method, ${ }^{19}$ by measuring the dielectric constant and the refractive index instead of the density. It is important to clarify the adequancy of approximations in each procedure.

\section{EXPERIMENTAL}

\section{Apparatus for Dielectric Measurements}

A three-terminal cell with main, counter and guard electrodes for a liquid was constructed following the report by Hartshown et al. ${ }^{20}$ The capacitance cell with a guard electrode is ade- quate to get higher accuracy in measurements of smaller capacitances (with air, vacuum, and non-polar organic solvent), because of the adequate screening of the stray capacitance of leads or solid insulators. The cell consists of platinum electrodes of two coaxial cylinders and double-walled cylindrical Pyrex glass as shown in Figure 1. The outer platinum cylinder having an inner diameter $r_{2}=21.0 \mathrm{~mm}$ is divided by circumferential narrow gaps into three parts, the central portion (A) with a length $L=60.0 \mathrm{~mm}$ forming the main electrode and two end portions (C), each about $8.3 \mathrm{~mm}$ in length, serving as the guard electrode. The inner platinum cylinder (B) $84.0 \mathrm{~mm}$ long is the counter electrode having an outer diameter $r_{1}=18.4 \mathrm{~mm}$. The cell capacitance in vacuum was calculated as $C_{\mathrm{v}}=25.3 \mathrm{pF}$ using the relation, $C_{\mathrm{v}}=0.2416 L / \log \left(r_{2} / r_{1}\right)(\mathrm{pF}) .^{21}$

Capacitance measurement was made on a cell with a capacitance bridge (General Radio

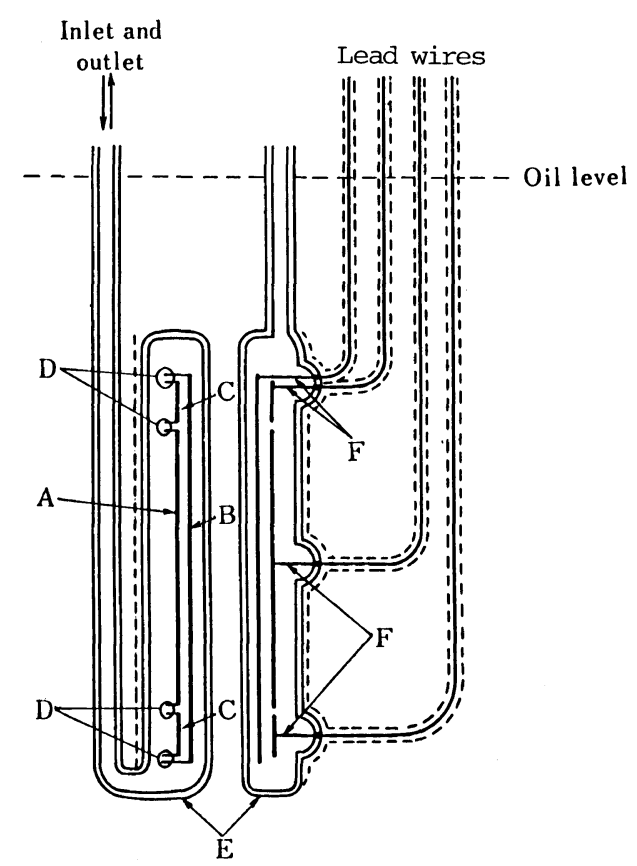

Figure 1. Three-terminal cell. A, B, and C, main, counter and guard platinum electrodes of cylindrical form; D, glass beads; E, a double-walled cylindrical Pyrex glass cell; F, platinum lead wires. The dotted lines indicate screens. 
Co., Type 1620) at a frequency of $10 \mathrm{kHz}$. The bridge is a type of transformer-ratio bridge that uses a single decade of a transformer voltage and multiple, fixed standard capacitors to provide six decades of resolution in capacitance. The cell capacitance with air $C_{0}$ was obtained as, $C_{0}=26.5650 \pm 0.0005 \mathrm{pF}$ at $25.0^{\circ} \mathrm{C}$. The observed value was consistent with the calculated one. The constancy of the cell capacitance measurements was found to be satisfactory through the course of the measurements over a month in which the cell was cleaned with benzene and evacuated and dried by the passage of dry air in each case. In these measurements, the cell was shielded by surrounding it with a stainless mesh and immersed in a silicone oil bath where the temperature was thermostatically controlled to $\pm 0.01^{\circ} \mathrm{C}$. The deviation of the cell capacitance from the mean value was \pm 2 parts in $10^{5}$.

\section{Materials}

Benzene and cyclohexane were repeatedly purified by distillation. The solvents were dried by passage through a molecular sieve $4 \mathrm{~A}$ prior to each distillation. Poly ( $p$-methoxystyrene) (PMeOSt) was prepared by radical polymerization by the use of benzoyl peroxide as the initiator at $80^{\circ} \mathrm{C}$. The crude product of PMeOSt was purified by reprecipitation using benzene and methanol and dried in vacuum. The number average molecular weight of the PMeOSt was $4.1 \times 10^{4}$, as determined by osmotic pressure measurements of toluene solutions with a Hewlett-Packard high speed membrane osmometer, Type 502.

\section{Measurements of Dielectric Constant, Density, and Refractive Index}

The capacitances of the cell with benzene and cyclohexane were measured by using the capacitance bridge at a frequency of $10 \mathrm{kHz}$ over the temperature range from 25 to $55^{\circ} \mathrm{C}$. The dielectric constants $\varepsilon$ relative to vacuum, i.e., not to air, were calculated by the relation,
$\varepsilon=C_{\mathrm{s}} /\left(C_{0} / \varepsilon_{\mathrm{air}}\right)$, where $C_{\mathrm{s}}$ is the capacitance of the cell with the solvent, $C_{0}$ is the cell capacitance with air and $\varepsilon_{\text {air }}$ is the dielectric constant of air $\left(\varepsilon_{\text {air }}=1.0005364\right.$ at $\left.20^{\circ} \mathrm{C}^{22}\right)$. The dielectric constants of the benzene solutions of PMeOSt were determined in a similar manner as above at a frequency of $10 \mathrm{kHz}$ at 25 and $40^{\circ} \mathrm{C}$. The concentrations of solutions were in the range of 1.9 to 5.5 weight percent. The densities of the benzene solutions of PMeOSt were determined with a flask type capillary pycnometer of $10 \mathrm{ml}$ in volume at 25 and $40^{\circ} \mathrm{C}$, from which the partial molar volumes of the polymer at each temperature were estimated. The refractive indices of the solutions were obtained at $546 \mathrm{~nm}$ wavelength using a Shimadzu differential refractometer at 25 and $40^{\circ} \mathrm{C}$.

\section{RESULTS AND DISCUSSION}

\section{Dielectric Constants of Benzene and Cyclo- hexane}

The dielectric constants $\varepsilon$ for benzene and cyclohexane are plotted against temperature in Figure 2. In the calculation of $\varepsilon$ with the

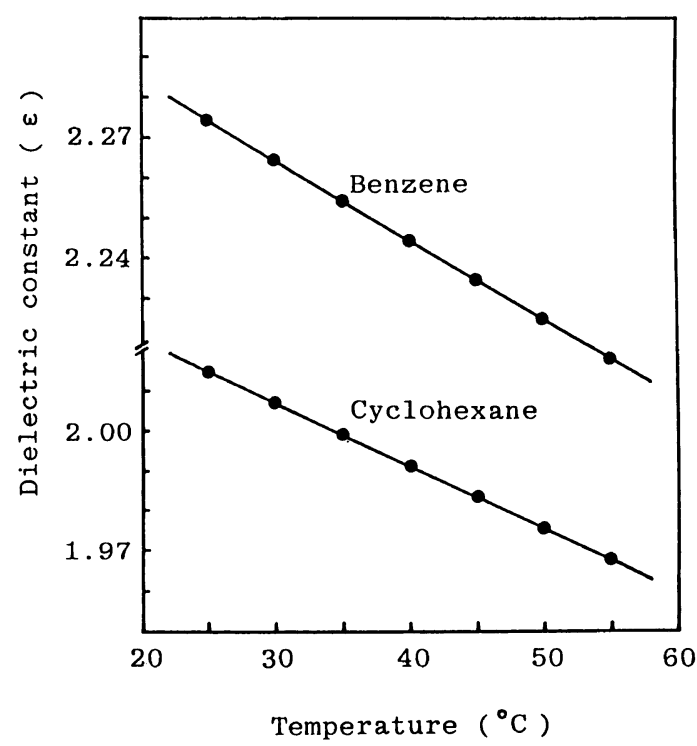

Figure 2. Plots of dielectric constant $\varepsilon v s$. temperature for benzene and cyclohexane. 
relation, $\varepsilon=\varepsilon_{\text {air }} \cdot C_{\mathrm{s}} / C_{0}$, the values of $\varepsilon_{\text {air }}$ and $C_{0}$ were assumed to be constant. These assumptions should be rather adequate, because the temperature dependence of $\varepsilon_{\text {air }}$ should be negligibly small and the change in the $C_{0}$ value was actually found to be negligibly small over the temperature range from 25 to $55^{\circ} \mathrm{C}$. As shown in Figure 2, linear relationships were obtained between $\varepsilon$ and temperature $t$ for benzene and cyclohexane. The relations obtained by the least-squares method are as follows,

$$
\begin{array}{r}
\varepsilon=2.2741_{0}-0.00197(t-25) \\
\text { for benzene } \\
\varepsilon=2.0147_{7}-0.00157(t-25) \\
\text { for cyclohexane } .
\end{array}
$$

In Table I, the observed values of $\varepsilon$ at 25 and $30^{\circ} \mathrm{C}$ and the temperature coefficient $\mathrm{d} \varepsilon / \mathrm{d} t$ for both solvents are listed. In Table II, the values of $\varepsilon$ and $\mathrm{d} \varepsilon / \mathrm{d} t$ reported for benzene and cyclohexane $e^{20,23-25}$ are shown. Studies on accurate measurements of dielectric constant for or-

Table I. Dielectric constants and temperature coefficients of benzene and cyclohexane

\begin{tabular}{llll}
\hline & \multicolumn{3}{c}{$\varepsilon$} \\
\cline { 2 - 3 } Solvent & $25^{\circ} \mathrm{C}$ & $30^{\circ} \mathrm{C}$ & $-\mathrm{d} \varepsilon / \mathrm{d} t$ \\
\hline Benzene & $2.2741_{0}$ & $2.2642_{5}$ & 0.00197 \\
Cyclohexane & $2.0147_{7}$ & $2.0069_{2}$ & 0.00157 \\
\hline
\end{tabular}

ganic solvents are rather few. Hartshown et al. ${ }^{20}$ carried out dielectric measurements of organic solvents with an objective of providing values of higher accuracy than had been available. The measurements were performed on well purified, i.e., carefully dried and moisture free, solvents such as benzene, cyclohexane etc. by using the apparatus composed of a Schering bridge and a three-terminal cell at a frequency covering the range $50 \mathrm{~Hz}$ to $10 \mathrm{kHz}$ at several temperatures between 20 and $30^{\circ} \mathrm{C}$. The Office of Standard Reference Materials of the National Bureau of Standards (NBS) has recently reported the standard reference materials for use in the calibration of cells for determination of dielectric constants, in which cyclohexane is the standard with a small value of $\varepsilon .{ }^{25}$ The dielectric constant of cyclohexane was measured by using a capacitance bridge (General Radio Co., Type 1615-A) with the three-terminal cell at a frequency of 0.75 to $12 \mathrm{kHz}$ over the temperature range of 10 to $40^{\circ} \mathrm{C}$. The maximum deviation of observed values from the mean in the measurements is listed as \pm 0.00004 . These results are shown in Table II.

Our results for the dielectric constants and temperature coefficients for benzene and cyclohexane agree well with those reported in the literature. The standard deviation calculated from the values obtained in three independent measurements of freshly purified benzene at

\begin{tabular}{|c|c|c|c|c|c|}
\hline \multirow{2}{*}{ Solvent } & \multicolumn{3}{|c|}{$\varepsilon$} & \multirow{2}{*}{$-\mathrm{d} \varepsilon / \mathrm{d} t$} & \multirow{2}{*}{ Reference } \\
\hline & $20^{\circ} \mathrm{C}$ & $25^{\circ} \mathrm{C}$ & $30^{\circ} \mathrm{C}$ & & \\
\hline \multirow{3}{*}{ Benzene } & $2.282_{6}$ & $2.272_{5}$ & $2.262_{8}$ & $0.0019_{8}$ & 23 \\
\hline & 2.284 & 2.274 & 2.264 & 0.0020 & 24 \\
\hline & 2.2836 & 2.2741 & 2.2646 & 0.00190 & 20 \\
\hline \multirow{3}{*}{ Cyclohexane } & 2.023 & 2.015 & 2.007 & 0.0016 & 24 \\
\hline & 2.0250 & 2.0173 & 2.0095 & 0.00155 & 20 \\
\hline & 2.02280 & 2.01517 & 2.00730 & 0.00154 & 25 \\
\hline
\end{tabular}
$25^{\circ} \mathrm{C}$ was \pm 0.00005 . These facts suggest that the dielectric measurements in the present

Table II. Lists of dielectric constants and temperature coefficients of benzene and cyclohexane 
study were carried out satisfactorily. The results also suggest that the dielectric constant of organic solvent with a small value of $\varepsilon$ can be obtained without using any standard substance as the ratio of the capacitance of the cell filled with the sample solvent and that of the empty cell.

\section{Dipole Moment of Poly(p-methoxystyrene)}

The dielectric constant $\varepsilon$, density $\rho$, and refractive index $n$ observed for the benzene solutions of PMeOSt at 25 and $40^{\circ} \mathrm{C}$ are

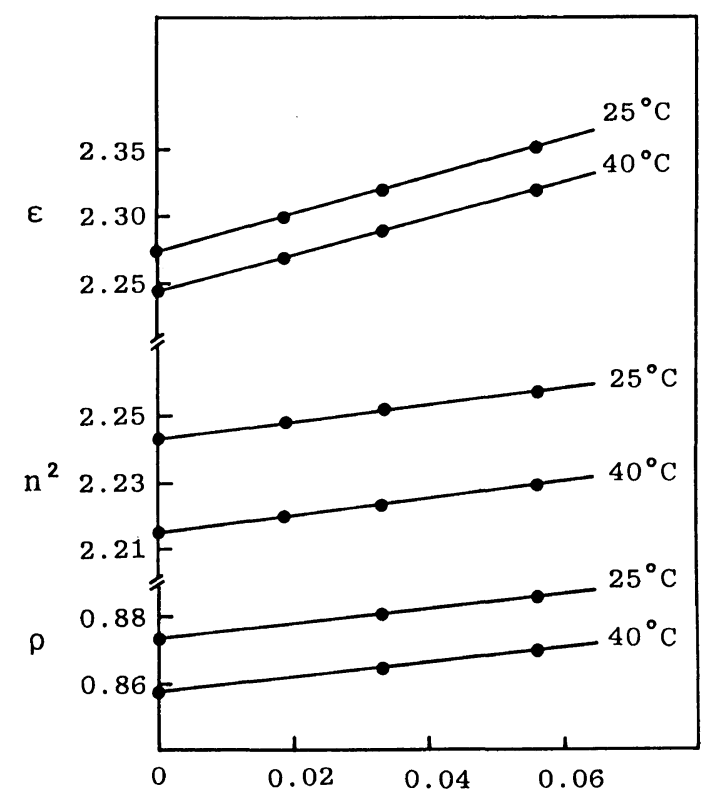

w

Figure 3. Plots of dielectric constant $\varepsilon$, refractive index squared $n^{2}$, and density $\rho$ against weight fraction $w$ for poly ( $p$-methoxystyrene) in benzene at 25 and $40^{\circ} \mathrm{C}$. shown in Table III. Plots of $\varepsilon, \rho$, and $n^{2}$ against the weight fraction $w$ gave linear relations as shown in Figure 3.

The dipole moment $\mu$ of a polar solute in a non-polar solvent is usually determined in two different procedures, both of which are based on Debye's theory for dielectric polarization. ${ }^{18}$ According to the theory, the molecular polarization of a solute $P_{2}$ is given by:

$$
\begin{aligned}
& P_{2}=\frac{\varepsilon-1}{\varepsilon+2} \frac{M}{\rho}=\frac{4 \pi N}{3}\left(\gamma+\frac{\mu^{2}}{3 k T}\right) \\
& P_{2}=P_{\mathrm{O}}+P_{\mathrm{E}}+P_{\mathrm{A}} \\
& P_{\mathrm{O}}=\frac{4 \pi N}{9 k T} \mu^{2}, \quad P_{\mathrm{E}}+P_{\mathrm{A}}=\frac{4 \pi N}{3} \gamma
\end{aligned}
$$

where, $\varepsilon$ and $\rho$ are the dielectric constant and density, $M$ is the molecular weight, $\gamma$ is the polarizability, $P_{\mathrm{O}}, P_{\mathrm{E}}$, and $P_{\mathrm{A}}$ are the orientation, electronic, and atomic polarizations, respectively, $k$ is the Boltzmann constant, $N$ is the Avogadro's number and $T$ is temperature.

For the determination of $\mu$, the orientation polarization $P_{\mathrm{O}}$ must be known. One of the procedures is a conventional method, that is, the molecular polarization $P_{2}$ is determined by the dielectric constant and the density of the polymer solution and $P_{\mathrm{O}}$ is calculated by subtracting $P_{\mathrm{E}}$ and $P_{\mathrm{A}}$ from $P_{2}$.

The specific polarization of the solution of a polar solute in a non-polar solvent $p_{12}$ is expressed by using the weight fraction $w$ of the solute as follows,

$$
p_{12}=\frac{\varepsilon_{12}-1}{\varepsilon_{12}+2} \frac{1}{\rho_{12}}=(1-w) p_{1}+w p_{2}
$$

\begin{tabular}{|c|c|c|c|c|c|c|}
\hline \multirow{2}{*}{$w$} & \multicolumn{3}{|c|}{$25^{\circ} \mathrm{C}$} & \multicolumn{3}{|c|}{$40^{\circ} \mathrm{C}$} \\
\hline & $\varepsilon$ & $\rho$ & $n$ & $\varepsilon$ & $\rho$ & $n$ \\
\hline 0 & 2.2741 & 0.87368 & 1.4979 & 2.2446 & 0.85762 & 1.4883 \\
\hline 0.018967 & 2.3012 & - & 1.4995 & 2.2702 & - & 1.4900 \\
\hline 0.033330 & 2.3211 & 0.88102 & 1.5007 & 2.2893 & 0.86515 & 1.4912 \\
\hline 0.056135 & 2.3539 & 0.88615 & 1.5025 & 2.3207 & 0.87052 & 1.4932 \\
\hline
\end{tabular}

Table III. Dielectric constants, densities, and refractive indices of poly( $p$-methoxystyrene) in benzene solution at 25 and $40^{\circ} \mathrm{C}$ 
where

$$
p_{1}=\frac{\varepsilon_{1}-1}{\varepsilon_{1}+2} \frac{1}{\rho_{1}} \text { and } p_{2}=\frac{\varepsilon_{2}-1}{\varepsilon_{2}+2} \frac{1}{\rho_{2}}
$$

in which suffixes 1,2 , and 12 refer to the solvent, solute and solution, respectively. The specific polarization of the solute $p_{2}$ is evaluated by using Halverstadt-Kumler equation $^{26}$ in place of the direct extrapolation by eq 2

$$
p_{2}=p_{1}\left(1+\frac{3 \alpha}{\left(\varepsilon_{1}-1\right)\left(\varepsilon_{1}+2\right)}-\frac{\beta}{\rho_{1}}\right)
$$

where $\alpha$ and $\beta$ are the specific increments at infinite dilution as,

$$
\alpha=\frac{\mathrm{d}\left(\varepsilon_{12}-\varepsilon_{1}\right)}{\mathrm{d} w}
$$

and

$$
\beta=\frac{\mathrm{d}\left(\rho_{12}-\rho_{1}\right)}{\mathrm{d} w}
$$

The molecular polarization $P_{2}$ is obtained by the relation,

$$
P_{2}=p_{2} \cdot M
$$

The electronic polarization $P_{\mathrm{E}}$ is equal to the molecular refraction found for the light of infinite wavelength, so that $P_{\mathrm{E}}$ is a quantity possible to determine experimentally. There is no appropriate method to determine $P_{\mathrm{A}}$. In general, $P_{\mathrm{A}}$ is small compared with $P_{\mathrm{E}},{ }^{27}$ except for a molecule whose atoms or atom groups are easily displaced relative to each other in the presence of a field. ${ }^{28}$ Therefore, $P_{\mathrm{A}}$ has been neglected or roughly estimated as $5-15 \%$ of the related $P_{\mathrm{E}}$. One plausible assumption is that the molecular refraction for the D-line is taken as the sum of $P_{\mathrm{E}}$ and $P_{\mathrm{A}}$,

$$
P_{\mathrm{E}}+P_{\mathrm{A}}=\frac{n_{\mathrm{D}}^{2}-1}{n_{\mathrm{D}}^{2}+2} \frac{M}{\rho}=[\mathrm{MR}]_{\mathrm{D}}
$$

where $n_{\mathrm{D}}$ is the refractive index of the solute for the D-line. $[\mathrm{MR}]_{\mathrm{D}}$ is a little larger than the molecular refraction for infinite wavelength.
The value of $[M R]_{D}$ is generally estimated as the sum of the appropriate atomic or bond refractions of the constituent atoms or bonds. By the use of the approximation, the dipole moment is expressed by:

$$
\begin{aligned}
\mu & =\left\{\frac{9 k T}{4 \pi N}\left(P_{2}-[\mathrm{MR}]_{\mathrm{D}}\right)\right\}^{1 / 2} \\
& =0.01281 \sqrt{\left(P_{2}-[\mathrm{MR}]_{\mathrm{D}}\right) T}
\end{aligned}
$$

This procedure, hereafter, is refered to as the DHK method.

Another procedure is the one proposed by Guggenheim and Smith $^{19}$ in which the dipole moment is determined by measuring the dielectric constant and refractive index of the solutions. Debye's equation is written for a dilute solution of a polar solute in a non-polar solvent with mole fraction $x$ as follows,

$$
\begin{aligned}
& \frac{\varepsilon_{12}-1}{\varepsilon_{12}+2} V_{12}=\frac{\varepsilon_{1}-1}{\varepsilon_{1}+2} V_{1}(1-x)+x P_{2} \\
& \frac{n_{12}^{2}-1}{n_{12}^{2}+2} V_{12}=\frac{n_{1}^{2}-1}{n_{1}^{2}+2} V_{1}(1-x)+x P_{\mathrm{E}}
\end{aligned}
$$

where, $V_{12}$ is the mean molar volume calculated by the relation, $V_{12}=(1-x) V_{1}+x V_{2}$, $V_{1}$ and $V_{2}$ are the partial molar volumes of the solvent and solute, respectively. If the atomic polarization of the solute is assumed to be given by the fictitious atomic polarization,

$$
P_{\mathrm{A}}=\left(\frac{\varepsilon_{1}-1}{\varepsilon_{1}+2}-\frac{n_{1}^{2}-1}{n_{1}^{2}+2}\right) V_{2}
$$

the equation for the orientation polarization is derived as follows,

$$
P_{\mathrm{O}}=\frac{3 M}{\rho_{1}}\left(\frac{\alpha}{\left(\varepsilon_{1}+2\right)^{2}}-\frac{v}{\left(n_{1}^{2}+2\right)^{2}}\right)
$$

where, $\alpha$ is given by eq 4 and $v$ is defined by:

$$
v=\frac{\mathrm{d}\left(n_{12}^{2}-n_{1}^{2}\right)}{\mathrm{d} w}
$$

The dipole moment $\mu$ is expressed as:

$$
\mu^{2}=\frac{27 k T M}{4 \pi N \rho_{1}}\left(\frac{\alpha}{\left(\varepsilon_{1}+2\right)^{2}}-\frac{v}{\left(n_{1}^{2}+2\right)^{2}}\right)
$$


This procedure is refered to as the G-S method.

The specific increments at infinite dilution $\alpha$, $\beta$, and $v$ were obtained from plots of $\varepsilon, \rho$, and $n^{2}$ against $w$, respectively, and the resulting lines were located by least-squares analysis (see Figure 3). The orientation polarization $P_{0}$ and dipole moment $\mu$ were estimated in the following manner. In the DHK method, the molecular polarization $P_{2}$ was calculated from eq 3 and 6 with $\alpha$ and $\beta$, and $[\mathrm{MR}]_{\mathrm{D}}$ was estimated from the bond refractions of the constituent bonds. ${ }^{22} \quad P_{\mathrm{O}}$ was obtained as the difference between $P_{2}$ and $[\mathrm{MR}]_{\mathrm{D}}$. $\mu$ was determined by eq 8 . In the G-S method, $P_{\mathrm{O}}$ and $\mu$ were calculated from eq 12 and 14, respectively, using the values of $\alpha$ and $v$. The average dipole moments per repeat unit for $\operatorname{poly}(p$ methoxystyrene) in benzene were obtained as $1.214\left(25^{\circ} \mathrm{C}\right), 1.221\left(40^{\circ} \mathrm{C}\right)$ by the DHK method and $1.204\left(25^{\circ} \mathrm{C}\right), 1.209\left(40^{\circ} \mathrm{C}\right)$ by the G-S method (in debye unit). The results are summarized in Table IV.

The values of $\mu$ for PMeOSt in benzene previously reported in the literature are 1.21 $\left(20^{\circ} \mathrm{C}\right),{ }^{6} 1.24\left(50^{\circ} \mathrm{C}\right),{ }^{6} 1.25\left(25^{\circ} \mathrm{C}\right),{ }^{13}$ and 1.17 $\left(25^{\circ} \mathrm{C}\right)^{16}$ (in debye unit). The results of the present study are very close to the values of $1.21\left(20^{\circ} \mathrm{C}\right)$ and $1.24\left(50^{\circ} \mathrm{C}\right)$ which were previously reported by one of the authors. The values of $\mu$ obtained in the present study are considered to be correct to \pm 2 parts in $10^{3}$, since the measurements of $\varepsilon, \rho$, and $n$ can be done very accurately and errors in these values were less than \pm 2 parts in $10^{4}$, respectively.

The results show that the $\mu$ values of $\mathrm{PMeO}$ St obtained by the two different methods were in fair agreement at the respective temperatures of 25 and $40^{\circ} \mathrm{C}$ and increased slightly with increase in temperature. Closer examination of the results indicated that $\mu$ obtained by the DHK method is about $1 \%$ larger than the one by the G-S method, which is more than the experimental error. We will now discuss the differences in $\mu$ values.

As mentioned before, in the DHK method, the sum of electronic and atomic polarization is assumed to be equal to the molecular refraction for the D-line and it is generally calculated as the summation of the appropriate atomic or bond refractions ${ }^{22}$ of the constituent atoms or bonds. As the molecular refraction is an additive and constitutive property, the summation seems to be reasonable

Table IV. Results for dipole moments of poly( $p$-methoxystyrene) in benzene at 25 and $40^{\circ} \mathrm{C}$

\begin{tabular}{|c|c|c|c|c|c|c|c|}
\hline \multirow{2}{*}{$\frac{\text { Temp }}{{ }^{\circ} \mathrm{C}}$} & \multirow{2}{*}{$\alpha$} & \multirow{2}{*}{$\beta$} & \multirow{2}{*}{$v$} & \multicolumn{2}{|c|}{ DHK method } & \multicolumn{2}{|c|}{ G-S method } \\
\hline & & & & $P_{\mathrm{O}} / \mathrm{ml}$ & $\mu$ (debye) & $P_{\mathrm{o}} / \mathrm{ml}$ & $\mu$ (debye) \\
\hline 25 & 1.4204 & 0.22202 & 0.24288 & 30.116 & $1.213_{9}$ & 29.610 & $1.204_{0}$ \\
\hline 40 & 1.3542 & 0.22952 & 0.25928 & 29.020 & $1.221_{2}$ & 28.430 & $1.209_{1}$ \\
\hline
\end{tabular}

Table V. Electronic, Atomic, and molecular polarizations of poly ( $p$-methoxystyrene) in benzene

\begin{tabular}{|c|c|c|c|c|c|}
\hline \multirow{2}{*}{$\frac{\text { Temp }}{{ }^{\circ} \mathrm{C}}$} & \multicolumn{2}{|c|}{ DHK method } & \multicolumn{3}{|c|}{ G-S method } \\
\hline & {$[\mathrm{MR}]_{\mathrm{D}} / \mathrm{ml}$} & $P_{2} / \mathrm{ml}$ & $P_{\mathrm{E}} / \mathrm{ml}^{*}$ & $P_{\mathrm{A}} / \mathrm{ml}^{*}$ & $P_{2} / \mathrm{ml}^{*}$ \\
\hline 25 & 39.856 & 69.972 & 39.998 & 0.488 & 69.951 \\
\hline 40 & 39.856 & 68.876 & 39.951 & 0.475 & 68.681 \\
\hline
\end{tabular}

* The partial molar volumes of poly( $p$-methoxystyrene) in benzene used to calculate the polarizations with an asterisk were calculated from the density of the polymer solution as $V_{2}=115.13 \mathrm{ml}$ at $25^{\circ} \mathrm{C}$ and $115.10 \mathrm{ml}$ at $40^{\circ} \mathrm{C}$. 
but the $[M R]_{D}$ value is altered slightly depending on whether it is calculated from the atomic refraction or bond refraction. This means that in the DHK method, it is unavoidable to bring some uncertainty into a final dipole moment. The molecular refraction for the structural unit of PMeOSt is calculated as $39.604 \mathrm{ml}$ from the atomic refraction and $39.856 \mathrm{ml}$ from the bond refraction. This small difference brings a difference of 0.005 debye into the $\mu$ value. $\mu$ in Table IV were calculated with $[\mathrm{MR}]_{\mathrm{D}}$ value obtained from the bond refraction. In the G-S method, $P_{\mathrm{E}}$ is the molecular refraction of a solute as similar as in the DHK method, but it was estimated from eq 10 by using the experimental data of the refractive index and the partial molar volume. $P_{\mathrm{A}}$ was obtained from eq 11 with $\varepsilon$ and $n$ for the solvent and the partial molar volume of the solute. These values are listed in Table $\mathrm{V}$, as well as the values of $P_{2}$ obtained by the DHK and the G-S methods.

$P_{\mathrm{E}}$ values obtained from eq 10 are very close to the $[\mathrm{MR}]_{\mathrm{D}}$ value calculated from the bond refractions. This agreement is rather reasonable, since the refractive index measurements for the estimation of $P_{\mathrm{E}}$ were made at the finite wavelength of $546 \mathrm{~nm}$, which is almost the same for the D-line. The G-S method is usually thought to be a fairly good approximation if the solvent used in the measurements has a small value of $\left(\varepsilon-n^{2}\right)$ such as benzene. $\left(\varepsilon-n^{2}\right)$ for benzene are 0.0304 at $25^{\circ} \mathrm{C}$ and 0.0296 at $40^{\circ} \mathrm{C}$. The $P_{\mathrm{A}}$ value assumed by eq 11 is a quantity proportional to $\left(\varepsilon-n^{2}\right)$ and is $0.488 \mathrm{ml}\left(25^{\circ} \mathrm{C}\right)$ and $0.477 \mathrm{ml}\left(40^{\circ} \mathrm{C}\right)$ for PMeOSt in benzene, which correspond to $c a$. 0.01 debye unit in $\mu$. It is evident that the slight disagreement between the $\mu$ values obtained by the two different procedures resulted from the different approaches to estimate $P_{\mathrm{E}}$ and $P_{\mathrm{A}}$. The exact value of $P_{\mathrm{E}}$ is able to be estimated from eq 10 by using the refractive index at infinite wavelength instead of the widely used method of obtaining it with the refractive index at a finite wavelength, while the esti- mation of $P_{\mathrm{A}}$ is not exact but based on a fictitious assumption in both the DHK and the G-S methods. Therefore, it is unavoidable that the uncertainty arising from the fictitious assumptions of $P_{\mathrm{A}}$ is brought into the final value of $\mu$, even if the exact value of $P_{\mathrm{E}}$ is obtained.

Fortunately, $P_{\mathrm{A}}$ for many substances is not large compared to the related $P_{\mathrm{E}}$, so both procedures can give appropriate results in the study on comparison of dipole moments of series of molecules in a given solvent or in the study on temperature dependence in a given solvent. Nevertheless, in the study on solvent effect of $\mu$, it should be noted that $P_{\mathrm{A}}$ assumed in the G-S method is a solvent dependent quantity.

Acknowledgment. This research was partially supported by a Grant-in-Aid for Scientific Research (No. 57470075), from the Ministry of Education, Science, and Culture of Japan.

\section{REFERENCES}

1. J. Marchal and H. Benoit, J. Polym. Sci., 23, 223 (1957).

2. W. H. Stockmayer, Pure Appl. Chem., 15, 539 (1967).

3. M. Shima, J. Polym. Sci., 56, 213 (1962).

4. A. Kotera, M. Shima, N. Yamaguchi, and T. Kobayashi, Bull. Chem. Soc. Jpn., 35, 1117 (1962).

5. M. Shima and A. Kotera, Makromol. Chem., 64, 172 (1963).

6. A. Kotera, K. Suzuki, K. Matsumura, M. Shima, and E. Joko, Bull. Chem. Soc. Jpn., 39, 750 (1966).

7. A. Kotera, K. Shimomura, and M. Shima, J. Polym. Sci., C, No. 30, 233 (1970).

8. T. Hirai, P. H. Khank, K. Tsuji, A. Sato, T. Tsuruta, A. Abe, T. Shimozawa, A. Kotera, N. Yamaguchi, and S. Kitahara, Polym. J., 11, 905 (1979).

9. N. Yamaguchi, M. Sato, E. Ogawa, and M. Shima, Polymer, 22, 1464 (1981).

10. M. Shima, N. Yamaguchi, M. Sato, and E. Ogawa, Eur. Polym. J., 19, 601 (1983).

11. P. J. Flory, "Statistical Mechanics of Chain Molecules," Interscience Publishers, New York, 1969.

12. L. L. Burshtein and T. P. Stepanova, Polym. Sci. U.S.S.R., 11, 2885 (1969).

13. B. Bayasal, B. A. Lowry, H. Yu, and W. H. Stockmayer, "Dielectric Properties of Polymers," F. 
E. Karasz, Ed., Plenum, New York, 1972, p 329.

14. F. Blasco and E. Riande, J. Polym. Sci., Polym. Phys. Ed., 21, 835 (1983).

15. A. E. Tonelli and L. A. Belfiore, Macromolecules, 16, 1740 (1983).

16. B. Baysal and L. Aras, Macromolecules, 18, 1693 (1985).

17. L. E. Mark, J. Chem. Phys., 56, 451 (1972); E. Saiz, J. E. Mark, and P. J. Flory, Macromolecules, 10, 967 (1977).

18. P. Debye, "Polar Molecules," Chemical Catalog, New York, 1929; P. Debye and F. Bueche, J. Chem. Phys., 19, 589 (1951).

19. E. A. Guggenheim, Trans. Faraday Soc., 45, 714 (1949); ibid., 47, 573 (1951); J. W. Smith, ibid., 46, 394 (1950).

20. L. Hartshown, J. V. L. Parry, and L. Essen, Proc. Phys. Soc., 68B, 422 (1955).

21. "A New Course in Experimental Chemistry," Vol. 5, Maruzen, 1976, Chapter 3.

22. "Handbook of Chemistry," Maruzen, 1984.
23. L. Hartshown and D. A. Oliver, Proc. Roy. Soc. London, Ser. A, 123, 664 (1929).

24. A. A. Maryott and E. R. Smith, "Tables of Dielectric Constants of Pure Liquids," NBS Circular, 1951, p 514.

25. C. G. Malmberg and M. G. Broadhurst, Catalog of NBS Standard Reference Materials, 1511, April (1969).

26. L. F. Halverstadt and W. D. Kumler, J. Am. Chem. Soc., 64, 2988 (1942).

27. R. J. W. Le Fèvre, "Dipole Moments," Methuen, London 1953; J. H. Van Vleck, "The Theory of Electric and Magnetic Susceptibilities," Oxford University Press, 1932; C. J. F. Böttcher and P. Bordewijk, "Theory of Electric Polarization," Elsevier Publishers, Co., 1978.

28. S. Dasgupta and C. P. Smith, J. Chem. Phys., 47, 2911 (1967); S. Sutton and J. E. Mark, J. Chem. Phys., 54, 5011 (1971); S. C. Liao and J. E. Mark, ibid., 59, 3825 (1973). 